\title{
A THEORETICAL PHOTOCATHODE EMITTANCE MODEL INCLUDING TEMPERATURE AND FIELD EFFECTS*
}

\author{
K. L. Jensen*, Naval Research Laboratory, Washington DC 20375 \\ D. W. Feldman, E. J. Montgomery, P. G. O'Shea, U of MD, College Park, MD 20742 \\ N. A. Moody, Los Alamos National Laboratory, Los Alamos, NM 87545 \\ J. Petillo, Science Applications International Corporation, Burlington, MA 01803
}

\begin{abstract}
A recently developed model of emittance \& brightness of photocathode based on "moments" of the electron emission distribution is extended here to non-zero temperatures and fields. Temperature impacts scattering and affects quantum efficiency. Fields affect emission probability and are important in the presence of low-work function coatings characteristic of cesiated dispenser photocathodes under development. Extensions of theoretical models are given, followed by analysis of their comparison with numerical simulations of the intrinsic emittance and brightness of a photocathode. The methodology is designed to facilitate development of photoemission models into comprehensive particle-in-cell (PIC) codes to address variation in surface coverage and topology.
\end{abstract}

\section{INTRODUCTION}

\section{Photocathodes}

Laser-switched photocathodes are widely used as the electron source of choice for prebunched beams needed by rf linacs, Free Electron Lasers (FELs) and accelerators. Consequently, the development of suitable high brightness photocathode sources to provide electron bunches with the requisite properties demanded by high power devices is an active area of research. The average power of the FEL is limited by characteristics of the electron beam: electrons outside the laser contribute less to the generation of coherent radiation and "halo" can cause damage. Moreover, higher current and smaller emittance enable shorter wavelength and more powerful FELs. Consequently, demands placed on the emittance and brightness of beams set constraints on what characteristics of the photocathode are allowable, as intrinsic emittance (that which originates at the cathode) cannot be mitigated elsewhere. It is the purpose of this work to utilize a distribution function, or Moments-based, approach that enables the calculation of emittance and brightness. Significantly, the Moments-based approach is in fact needed if the photoemission model is to be incorporated into advanced beam simulation codes that model vacuum electronic devices.

\section{MOMENTS}

\section{Definitions}

The moments of a distribution function, as with the classical definition, are given by finding averages of the powers of momenta with a distribution function. Averages of a quantity $O$ are given by

$$
\langle O\rangle \equiv \frac{\int d \mathbf{r} d \mathbf{k} O(\mathbf{r}, \mathbf{k}) f(\mathbf{r}, \mathbf{k})}{\int d \mathbf{r} d \mathbf{k} f(\mathbf{r}, \mathbf{k})}
$$

in terms of which the rms normalized emittance and brightness are given by

$$
\begin{aligned}
\varepsilon_{n, r m s}(z) & =\frac{\hbar}{m c} \sqrt{\left\langle x^{2}\right\rangle\left\langle k_{x}^{2}\right\rangle-\left\langle x k_{x}\right\rangle^{2}} \\
B_{n} & =\frac{2 I_{e}}{\pi^{2} \varepsilon_{n}^{2}}
\end{aligned}
$$

where $\hbar$ is Planck's constant over $2 \pi, m$ is the electron mass, $c$ the speed of light, $x$ and $k_{x}$ are position and momentum, and $I_{e}$ is the emitted electron current (not current density). The distribution function is defined by

$$
f(\vec{x}, \vec{k})=\Theta\left(\rho_{c}-\rho\right) f(E(\vec{k}))
$$

where $\Theta$ is the Heaviside step function, $\rho_{c}$ is the radius of the illumination (or emission) area, and $f$ is the distribution in energy of the emitted electrons. To calculate the emission distribution, the distribution of electrons within the photocathode are assumed to be Fermi-Dirac, and the transmission probability is taken to be a modified form of the usual WKB formulation. Therefore

$$
\begin{aligned}
D\left(E_{z}\right) & =\left(1+\exp \left[\beta_{F}\left(\mu+\phi-E_{z}\right)\right]\right)^{-1} \\
f_{F D}(E) & =\left(1+\exp \left[\beta_{T}(E-\mu)\right]\right)^{-1}
\end{aligned}
$$

where $D$ is the transmission probability, $E_{z}$ is the energy equivalent of the momentum directed into the barrier, $\mu$ and $\phi$ are the Fermi level and work function (reduced by Schottky barrier lowering, i.e., $\phi=\Phi-\sqrt{4 Q F}$, where $Q$ $=0.36 \mathrm{eV}-\mathrm{nm}$ is the coefficient of $1 / x$ in the image charge model), respectively, and $\beta_{T}$ and $\beta_{F}$ are energy slope factors, the former of which is $\beta_{T}=1 / k_{B} T$, where $k_{B}$ is Boltzmann's constant. Therefore, Moments are given by

$$
M_{n}\left(k_{s}\right) \equiv(2 \pi)^{-3} \int k_{s}^{n} f(E(\vec{k})) d^{3} k
$$

where $k_{s}$ is the momentum component of interest and where $s=z$ or $\rho$, in terms of which emittance is given by

$$
\varepsilon_{n, r m s}(x)=\left(\frac{\hbar}{2 m c}\right) \rho_{c} \sqrt{\frac{M_{2}\left(k_{\rho}\right)}{M_{0}}}
$$

If the emission distribution is given by a MaxwellBoltzman distribution, then it is a straightforward matter to show that Eq. 6 gives for thermal emittance 


$$
\left.\varepsilon_{n, r m s}(x)\right|_{\text {thermal }} \Rightarrow \frac{\rho_{c}}{2} \sqrt{\frac{k_{B} T}{m c^{2}}} .
$$

In contrast, in the case of photoemission, the distribution function is much more complex, and is

$$
\begin{gathered}
M_{n}\left(k_{s}\right)=(2 \pi)^{-3}\left(\frac{2 m}{\hbar^{2}}\right)^{3 / 2} \int_{0}^{\infty} E^{1 / 2} d E \int_{0}^{\pi / 2} \sin \theta d \theta \times \\
\left(k_{s}\right)^{n} D\left\{(E+\hbar \omega) \cos ^{2} \theta\right\} f_{\lambda}(\cos \theta, E+\hbar \omega) \times \\
f_{F D}(E)\left\{1-f_{F D}(E+\hbar \omega)\right\}
\end{gathered}
$$

where the Fermi Dirac distributions indicate occupancy of initial and final states, and where the scattering factor $f_{\lambda}$ is given by

$$
\begin{aligned}
f_{\lambda}(x, E) & =\frac{x}{x+p(E)} \\
p(E) & =\frac{m \delta}{\hbar k(E) \tau(E)}
\end{aligned}
$$

where $\delta$ is the laser penetration depth and the factor $p$ is the ratio between $\delta$ and the distance the electron travels between scattering events. The notation is similar to that found in our previous work [3] though the evaluation of the relevant terms - in particular, the relaxation time - has undergone substantial revision [4] and has been profitably used in the characterization of quantum efficiency from cesiated surfaces [5].

The methodology has been used elsewhere to find the corrections to the Fowler-Dubridge (FD) model of quantum efficiency (QE) [6], from which it was found that the FD function $U$ is replaced with

$$
P(\hbar \omega) \approx \frac{(\hbar \omega-\phi)^{2}+2 \zeta(2)\left(\beta_{T}^{-2}+\beta_{F}^{-2}\right)}{2 \hbar \omega(2 \mu-\hbar \omega)}
$$

where $\zeta(n)$ is the Riemann Zeta function. The Momentsbased approach was used to estimate the QE from a partially cesiated surface, and a comparison of its performance with experimental data is given in Fig. 1.

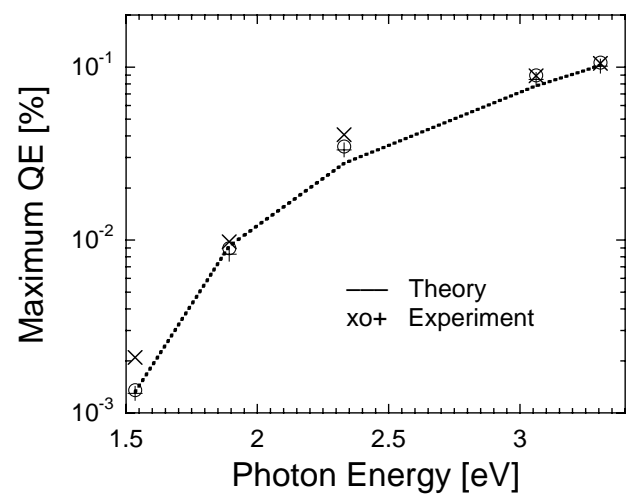

Figure 1: Comparison of theory to experiment for the maximum $\mathrm{QE}$ from a partially cesiated surface.

For standard conditions characteristic of photoemission, the energy slope factors $\beta_{T}$ and $\beta_{F}$ are large as both the transmission probability and the FermiDirac distribution function resemble step-functions. Therefore, Eq. 10 does anticipate the consequences of higher fields and temperatures.

\section{Evaluation of the Moments}

Equation 8 was evaluated for bare and cesiated metal conditions: copper was chosen as the bulk metal because it is used as a photomaterial, because it has received considerable attention both by itself and with coatings such as Cs, and because the impact of low work function coatings are thereby given perspective. The Moments were numerically evaluated and compared to an analytical model based on the leading order behavior of Eq. 8 given by

$$
\begin{aligned}
& M_{0} \approx \frac{\sqrt{2 \mu m^{3}}}{2(2 \pi)^{2} \hbar^{3}}\left\{\frac{(\hbar \omega-\phi)^{2}}{(\mu+\phi)[p(\hbar \omega+\mu)+1]}\right\} \\
& M_{2} \approx \frac{2 m \mu}{3 \hbar^{2}} \frac{(\hbar \omega-\phi)}{(\hbar \omega+\mu)} M_{0}
\end{aligned}
$$

from which we determine

$$
\begin{gathered}
\varepsilon_{n, \text { rms }}(\text { photo }) \approx \frac{\rho_{c}}{2}\left[\frac{\mu(\hbar \omega-\phi)}{3 m c^{2}(\hbar \omega+\mu)}\right]^{1 / 2} \\
B_{n} \approx\left(\frac{q}{\hbar \omega}\right)\left(\frac{2 m c^{2}}{\pi \mu}\right)\left(\frac{1-R(\omega)}{1+p(\mu+\hbar \omega)}\right) \times \\
{\left[\frac{(\hbar \omega+\mu)(\hbar \omega-\phi)(3 \mu+\phi-\hbar \omega)}{\hbar \omega(\mu+\phi)(2 \mu-\hbar \omega)}\right] I_{\lambda}}
\end{gathered}
$$

where $R$ is the reflectivity as a function of photon frequency, $I_{\lambda}$ is the incident laser intensity, $q$ is the unit charge, and other terms have their usual meanings. Observe that the limiting form of Eq. 12 does not exhibit the temperature and field dependence of Eq. 10 (apart from the Schottky factor in $\phi$ ) due to the approximation (see Eq. 4) of the transmission probability and supply function by step functions.

\section{RESULTS}

\section{Copper and Cesiated Copper}

Evaluations of the Moments were made for high temperature as a function of field, from which the numerical evaluation of photo-emittance was compared to the asymptotic form of Eq. 12 and shown in Figs. 2 and 3 for bare copper with a work function of $4.5 \mathrm{eV}$ and copper covered with a monolayer of cesium, for which the reduced work function was taken to be $1.8 \mathrm{eV}$. To highlight where deviation occurs, a large temperature was considered of $1600 \mathrm{~K}$ (for lower temperatures, the agreement between the asymptotic model and the numerical calculation is better). It is seen, therefore, that when the photon energy is comparable to the barrier height above the Fermi level, then the discrepancies between the analytical model and its numerical counterpart are more pronounced: note that the apparent agreement at high fields in Fig. 2 is because of the larger lowering of the barrier due to field, so that the difference between photon energy and barrier maximum is more pronounced and the agreement correspondingly better. From Fig. 3 however, it is clear that the magnitude of the 
work function matters (it impacts the transmission probability), as the cesiated surface has a substantially lower barrier than bare copper: under such conditions, the analogues of the field and temperature terms apparent in Eq. 10 make their presence known.

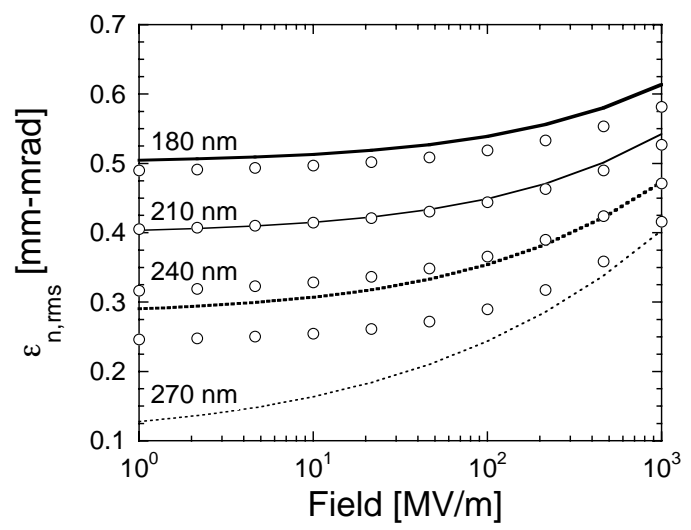

Figure 2: Comparison of the asymptotic and analytic equation for emittance (Eq. 12) with its numerical evaluation using Eq. 8 for bare copper conditions.

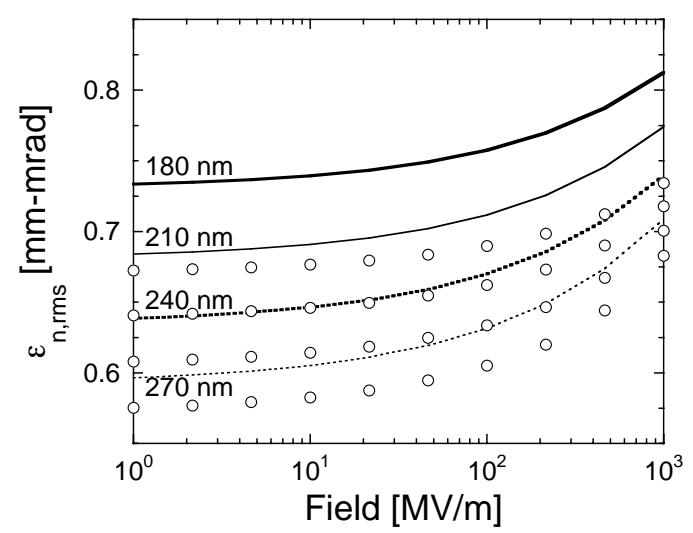

Figure 3: Comparison of the asymptotic and analytic equation for emittance (Eq. 12) with its numerical evaluation using Eq. 8 for a copper surface covered with a monolayer of cesium.

\section{Future Work}

First, the evaluation and comparison of analytical models of brightness to numerical models is complicated by its dependence upon reflectivity, laser penetration depth, and relaxation time, all of which depend on photon energy and the latter on laser intensity and duration. Such a comparison shall be reported separately.

Second, the methodology presented herein (especially the techniques developed for numerical evaluation) to investigate questions of photocathode emittance and brightness, are being adapted into numerical models of quantum efficiency for Particle-In-Cell codes such as
MICHELLE [2] and used to provide analogous models of "dark current" due to field and thermal emission. With such models in place, questions relating to the impact of variation in work function across the surface (arising from non-uniform coverage, differing crystal faces, and contamination [3,7]) or field enhancement (arising from bumps, scratches and protrusions) can be considered along the lines of a program described in Ref. [2].

\section{CONCLUSION}

An experimentally validated model of quantum efficiency based on a Moments approach was used to investigate photocathode emittance and brightness. An asymptotic model of the latter quantities on the assumption that the distribution functions and transmission probabilities inherent in the Moments are approximately step-function-like was given and compared to the numerical model. For bare metal parameters and moderate fields, the agreement between the numerical results and the analytical model is reasonably good. When the fields and temperatures are of a more dramatic nature, and especially when the work function has been significantly reduced by coatings such as Cesium, then discrepancies arise between the numerical and analytical models. The aim is to characterize cesium dispenser photocathodes under development and to provide the requisite emission models for PIC codes such as MICHELLE.

Acknowledgements: We gratefully acknowledge support provided by the Joint Technology Office and the Office of Naval Research.

\section{REFERENCES}

[1] K.L. Jensen, P.G. O'Shea, D.W. Feldman, N.A. Moody, Appl. Phys. Lett. 89, 224103 (2006).

[2] J. Petillo, D. Panagos, K. Jensen, B. Levush, "Detailed Photoemission Modeling Using The 3D Finite-Element PIC Code MICHELLE" (TUODC01, Particle Accelerator Conf. 2007, Albuquerque, NM).

[3] K.L. Jensen, D.W. Feldman, N.A. Moody, and P.G. O'Shea, J. Appl. Phys. 99, 124905 (2006).

[4] K.L. Jensen, D.W. Feldman, N.A. Moody, P.G. O'Shea, and E.J. Montgomery, Submitted to J. Appl. Phys. (2007).

[5] N.A. Moody, K.L. Jensen, D.W. Feldman, P.G. O'Shea, E.J. Montgomery, Appl. Phys. Lett. 90, 114108 (2007).

[6] K.L. Jensen, J. Appl. Phys. (accepted for pub.) (2007).

[7] D.H. Dowell, F.K. King, R.E. Kirby, J.F. Schmerge, and J.M. Smedley, PRST-AB 9, 063502 (2006). 